\title{
Issues of Development of Solar Collectors with High Efficiency
}

\author{
Mirsoli O. Uzbekov* and Bekzod B. Boynazarov \\ Fergana Polytechnic Institute \\ Republic of Uzbekistan, Fergana
}

Received 14.09.2021, received in revised form 21.10.2021, accepted 21.11.2021

\begin{abstract}
The article deals with the creation of energy-efficient solar water collectors.
In order to develop energy-efficient solar water collectors (SWC), methods of rational enhancement of heat transfer in the channels of the collectors are proposed. It is noted that the methods used to increase heat transfer from the collector walls to the water should be tailored to a low-speed flow of the heat carrier. With such flow regimes, the most acceptable ways to enhance heat transfer are: flow swirling, the use of artificial roughness, renewal of the hydrodynamic boundary layer through the creation of an alternating pressure gradient in the flow.

The analysis of the conducted research shows that the maximum effects of increasing heat transfer occur in the range of Re numbers from 2000 to 8000, i. e. in the laminar flow region and in the region of underdeveloped turbulence. An efficiency criterion is obtained for the conditions under which enhancers are installed inside the pipes. The criterion determines the ratio of the number of pipes with enhancers to the number of smooth pipes.

The calculation is carried out for cases in which the heating capacity, flow rate, power for heat carrier circulation and the pipe diameter of the SWC with smooth pipes are equal to the heating capacity, flow rate, power for heat carrier circulation and pipe diameter of the SWC with the pipes with enhancers. In addition to the specified efficiency criterion of the SWC, a heat transfer comparison formula of the SWC having pipes with enhancers and SWC with smooth pipes is obtained.
\end{abstract}

Keywords: solar water collectors, efficiency, heat transfer, heat engineering, convection, solar engineering. 


\title{
Вопросы разработки солнечных коллекторов \\ с высоким коэффициентом полезного действия
}

\author{
М. О. Узбеков, Б. Б. Бойназаров \\ Ферганский политехнический институт \\ Республика Узбекистан, Фергана
}

\begin{abstract}
Аннотация. В статье рассматриваются вопросы создания энергоэффективных солнечных водяных коллекторов.

Для разработки энергетически эффективных солнечных водяных коллекторов (СВК) предлагается использование методов рациональной интенсификации теплообмена в каналах коллекторов. Отмечено, что применяемые способы повышения теплопередачи от стенок коллектора к воде должны быть разработаны для условий низкоскоростного течения теплоносителя. При таких режимах течения наиболее приемлемыми способами интенсификации теплоотдачи можно считать: закрутку потока, применение искусственной шероховатости, обновление гидродинамического пограничного слоя за счет создания в потоке знакопеременного градиента давления.

Анализ проведенных исследований показывает, что наибольшие эффекты увеличения теплоотдачи возникают в области чисел Re от 2000 до 8000, т. е. в ламинарной области течений и области слабо развитой турбулентности. Для условий, при которых интенсификаторы теплообмена установлены внутри труб, получен критерий эффективности, определявший отношение числа труб с интенсификаторами к числу гладких труб.

Расчет выполнен для условий, когда теплопроизводительность, расход, мощность на прокачку теплоносителя и диаметр труб СВК с гладкими трубами равны теплопроизводительности, расходу, мощности на прокачку теплоносителя, диаметру труб СВК с интенсифицированными трубами. Кроме указанного критерия эффективности СВК получена формула сравнения теплообмена в СВК с интенсифицированными трубами и СВК с гладкими трубами.
\end{abstract}

Ключевые слова: солнечные водяные коллекторы, эффективность, теплообмен, теплотехника, конвекции, гелиотехника.

Цитирование: Узбеков, М.О. Вопросы разработки солнечных коллекторов с высоким коэффициентом полезного действия / М.О. Узбеков, Б. Б. Бойназаров // Журн. Сиб. федер. ун-та. Техника и технологии, 2021, 14(8). С. $942-949$. DOI: $10.17516 / 1999-494 X-0364$

Введение. Создание энергоэффективных солнечных водяных коллекторов (СВК) является одной из актуальных задач современной гелиотехники и теплотехники [1]. Для повышения эффективности СВК целесообразным считается применение методов рациональной интенсификации теплоотдачи, когда рост теплообмена соизмерим или опережает рост гидродинамического сопротивления. Анализ гидродинамических и температурных режимов показывает, что в традиционных гладкопластинчатых или гладкотрубных каналах коллекторов, как правило, формируется режим свободной конвекции, характеризующийся малыми скоростями теплоносителя $(\omega<0,1 \mathrm{M} / \mathrm{c})$. Такой гидродинамический режим малоэффективен с точки зрения теплопередачи, и, следовательно, традиционные СВК, работающие в таком режиме, имеют большие габариты [2].

Методы и материалы. Простое повышение скорости теплоносителя с помощью насосов приводит к неоправданному росту мощности, затрачиваемой насосом, так как

$$
N \approx \omega^{3} .
$$

$$
-943-
$$


Изучение способов интенсификации теплообмена, применяемых в промышленной теплотехнике и теплоэнергетике, показало, что большинство методов повышения теплоотдачи разработано для высоких скоростей теплоносителей, т. е. для турбулентной области течения. Учитывая, что теплообмен в СВК происходит в низкоскоростной области течения, наиболее приемлемыми можно считать следующие способы интенсификации теплоотдачи:

- закрутка потока;

- применение искусственной шероховатости;

- обновление гидродинамического пограничного слоя за счет создания в потоке знакопеременного градиента давления [1-5].

Также очевидно, что для организации интенсификации теплообмена в каналах СВК необходимо переходить в область смешанной конвекции, т. е. в область совместного действия свободной и вынужденной конвекций.

Первый способ интенсификации может быть организован путем тангенциального подвода теплоносителя либо с помощью шнеков или завихрителей потока. Данный метод заключается в использовании гидродинамических возможностей закрученного потока с целью интенсификации теплообмена.

Закрутка потока служит одним из эффективных способов гидродинамического воздействия на поток, при этом в трубе коллектора образуется движение теплоносителя сложной траектории. Так как движение воды в коллекторе имеет ламинарный характер, то в канале возникает закрученный поток, который при небольших числах Re является малоизученным в гелиотехнике. Закрученный поток может быть организован двумя путями. Первый закрученный поток с постоянным шагом, второй - с переменным шагом закрутки [6, 7].

Первый тип закрученного потока создается с помощью шнека, который размещен внутри трубы, второй тип закрутки может быть создан путем начальной закрутки и ее постепенным затуханием вдоль трубы коллектора. Этот тип потока создается при помощи внешнего завихрителя. Следует отметить, что применение шнеков приводит к удорожанию конструкции СВК и, соответственно, к увеличению массы коллектора.

Исследования показывают $[8,10]$, что наибольшие эффекты увеличения теплоотдачи возникают в области чисел Re от 2000 до 8000 , т. е. в ламинарной области течений и области слабо развитой турбулентности. Также отмечено, что применение любого из методов сопровождается ростом гидравлического сопротивления, для полной оценки эффективности способа интенсификации теплообмена наиболее целесообразным считается применение соотношения

$$
\left(N u / N u_{2 \pi}\right) /\left(\xi / \xi_{2 \pi}\right)=f(\mathrm{Re}) .
$$

К сожалению, отсутствие у ряда авторов опытных данных по гидравлическому сопротивлению не позволяет оценить эффективность всех типов поверхностей.

Следующий способ интенсификации теплообмена, который может быть приемлемым в каналах и трубах СВК, это искусственная шероховатость. Исследования теплообмена, проведенные при числах Re-10 $-10^{5}$, показали, что в трубах с дискретной шероховатостью, которые располагаются на внутренней поверхности, и отстоящих друг от друга на определенном расстоянии наблюдается значительное повышение теплообмена при одновременно существенном повышении гидравлического сопротивления. Такие трубы широко применяются в теплотехни-

$$
-944-
$$


ке и теплоэнергетике, а именно в конденсаторах паровых труб, подогревателях и других теплообменниках. Указанные выступы на трубах изготовляют накаткой роликами труб с помощью приспособления к токарному станку. При небольших значениях относительной шероховатости $\mathrm{Nu} / \mathrm{Nu} u_{2 л}>\xi / \xi_{2 л}$ т. е. рост теплообмена превышает рост гидравлического сопротивления. Основными геометрическими характеристиками шероховатости труб являются $\mathrm{d} /$ Д, t/h, t/Д, где $\mathrm{t}$ - шаг, $\mathrm{h}$ - высота выступа шероховатости, Д - диаметр трубы, $\mathrm{d}=$ Д-2h.

Опытами установлено, что интенсификация теплообмена наблюдается при числах $\operatorname{Re}=10^{4}$ и выше. При $\mathrm{t} / \mathrm{h}=7-8$ оторвавшийся поток присоединяется к поверхности и далее развивается турбилизированный пограничный слой, за каждым последующим элементом шероховатости процесс периодически повторяется. В пристенной области турбулентности $\mathrm{Tu}=15 \%$, что характерно для струйных течений.

К способу интенсификации теплообмена в трубах каналах может быть отнесен метод создания в потоке знакопеременного градиента давления. Применение последовательно чередующихся диффузорных и конфузорных участков с углами расширения $12^{0}$ в интервале $\mathrm{Re}=$ $(10-80) 10^{3}$ позволяет путем создания в потоке поперечных градиентов давления непрерывно замещать пограничный слой внешним потоком. Применение таких турбулизаторов для решения проблемы интенсивности обменных процессов за счет периодического механического отрыва пограничного слоя особенно эффективно в области относительно малых значений $\mathrm{Re}=$ $(3-20) 10^{3}$.

Известно, что энергетически выгодным считается режим турбулентного течения. Следовательно, не возмущая ядро потока, более эффективным гидродинамическим режимом считается режим, в котором в узкой пристенной области течения, состоящей из коротких псевдоламинарных пограничных слоев, осуществляется перенос тепла по законам турбулентного обмена.

Для получения такой структуры течения необходимым считается выполнение двух условий:

Первое - размещение турбулизаторов на поверхности теплообмена, или закручивателей потока.

Второе - обеспечение необходимой скорости ядра потока.

Оценки показывают, что при скорости потока $\omega=0,5$ м/с при диаметрах трубы СВК $\mathrm{d}=$ 15 мм и более число Рейнольдса потока достигает $10^{4}$. В этих случаях переход к турбулизированному пристенному потоку осуществляется достаточно легко.

В чередующемся диффузорно-конфузорном канале (диффузор имеет малый угол расширения) энергия турбулентности, накопленная в диффузоре, полезным образом используется в конфузоре. Так как течение жидкости или газа по диффузору сопровождается существенным увеличением коэффициента турбулентного обмена, следует ожидать заметной интенсификации теплообмена.

Протяженность диффузорных частей канала даже при малых углах расширения должна быть ограничена не только с целью уменьшения объема аппарата, но и для предотвращения крупномасштабных отрывов - эффекта, приводящего к увеличению энергетических потерь.

Визуальные исследования пристенных течений каналов диффузор-конфузор в области чисел $\operatorname{Re} 610^{3} \div 710^{4}$ показали, что направление потока вблизи стенок сужающегося и рас-

$$
-945-
$$


ширяющегося каналов может быть переменным в зависимости от угла раскрытия диффузора $\gamma$; отмечено, что при $\gamma=3,4^{0}$ жидкость вблизи стенок двигалась в том же направлении, что и основной поток. Однако при $\gamma=6,3^{0}$ обнаруживались небольшие области обратного течения непосредственно после начала расширяющегося участка, что указывает на существование отрыва потока. С увеличением угла $\gamma$ область обратного течения увеличивается и наиболее четкая картина обратного течения была получена при $\gamma=14,8^{0}$. Таким образом, микроотрывы потока от поверхности канала диффузор - конфузор способствуют непрерывному сокращению гидродинамического пограничного слоя и, как следствие, его уменьшению, тем самым интенсифицируя теплообмен.

Исходя из теории интенсификации теплообменных процессов, турбулизаторы потока должны обеспечивать существование первенства $N u / N u_{2 л}>\xi / \xi_{2 л}$, где $N u_{2 л}$ и $\xi_{2 л}$ - теплообмен и гидравлическое сопротивление гладких труб. В этом случае интенсификация теплообмена энергетически более выгодна.

Для турбулизаторов, выполненных в виде дискретной шероховатости или конфузорнодиффузорного профиля, важным считается их плавное обтекание, снижающее профильную составляющую гидродинамического сопротивления, которая увеличивает общее гидродинамическое сопротивление канала.

В настоящее время в теплотехнике и теплоэнергетике разработаны различные критерии энергетической оценки интенсифицированных поверхностей. Для закрученных потоков более правильным считается применение сравнительного анализа различных случаев закрученного потока по количеству тепла, переданному через рассматриваемую поверхность нагрева, при одинаковой затрате мощности на преодоление сопротивления движению жидкости в трубе. Для сравнения используется зависимость

$$
E=\frac{\alpha \Delta t}{N_{1}} \quad\left(N_{1}=\frac{\Delta P V}{F}\right)
$$

где $\alpha$ - средний коэффициент теплоотдачи $\mathrm{BT} / \mathrm{M}^{20} \mathrm{C} ; \mathrm{N}_{1}$ - мощность, затрачиваемая на преодоление сопротивления движению, включая сопротивление входа, и отнесенная к поверхности нагрева Вт/ $\mathrm{M}^{2}$. Сравнение проводится при $\mathrm{N}_{1}=\mathrm{idem}$ и $\Delta \mathrm{t}=\mathrm{idem}$. Другим способом оценки теплообменных поверхностей является сопоставление их по количеству теплоты, передаваемой в единицу времени Q; затрачиваемой мощности $\mathrm{N}$, площади поверхности теплообмена $\mathrm{F}$ :

$$
K_{Q}=\frac{Q^{\prime \prime}}{Q^{\prime}} ; \quad K_{N}=\frac{N^{\prime \prime}}{N^{\prime}} ; \quad K_{F}=\frac{F^{\prime \prime}}{F^{\prime}} .
$$

Решение задачи сводится, таким образом, к определению $\mathrm{K}_{\mathrm{Q}}$ при $\mathrm{K}_{\mathrm{N}}=1 ; \mathrm{K}_{\mathrm{N}}$ при $\mathrm{K}_{\mathrm{Q}}=1$ и $\mathrm{K}_{\mathrm{F}}=1 ; \mathrm{K}_{\mathrm{F}}$ при $\mathrm{K}_{\mathrm{Q}}=1 ; \mathrm{K}_{\mathrm{N}}=1$.

Следующим способом оценки эффективности теплообменной поверхности, заключающейся в сравнении площади поверхности теплообмена, является метод, предложенный в [2, 9]. Получим в соответствии с этим методом формулу сопоставления трубчатой поверхности СВК, т. е. определим уменьшение поверхности нагрева вследствие применения интенсифицированных труб вместо гладких. Расчет произведем для условий, когда теплопроизводительность, расход, мощность на прокачку теплоносителя и диаметр труб коллектора с гладкими трубами равны

$$
-946-
$$


производительности, расходу, мощности на прокачку теплоносителя и диаметру труб с интенсифицированными трубами. Поставленные условия приводят к следующим уравнениям:

$$
\begin{aligned}
& \xi \frac{\rho \omega^{3}}{8} \ell \pi d=\xi_{2 n} \frac{\rho \omega^{3}}{8} \ell_{2 n} \pi d_{2 n} \\
& \frac{\pi d^{2}}{4} \omega n=\frac{\pi d^{2}{ }_{2 \pi}}{4} \omega_{2 n} n_{2 n} \\
& \alpha \pi d \ell \Delta t n=\alpha_{2 n} \pi d_{2 n} \ell_{2 n} \Delta t_{2 n} n_{2 \pi} .
\end{aligned}
$$

Получим из

$$
\frac{n_{2 n}}{n}=\frac{\omega}{\omega_{2 n}}
$$

из (7), считая $\Delta \mathrm{t}=\Delta$ tгл, получим

$$
\frac{n}{n_{2 n}}=\frac{\alpha_{2 n} l_{2 n}}{\alpha \ell}
$$

из (5) получим

$$
\frac{\ell_{2 \pi}}{\ell}=\frac{\xi_{2 \pi}}{\xi} \frac{\omega_{2 \pi}{ }^{3}}{\omega^{3}}
$$

из (8) с учетом (9) получим

$$
\frac{n}{n_{2 \pi}}=\frac{\alpha_{2 n}}{\alpha} \frac{\xi^{2}}{\xi_{2 n}}\left(\frac{n_{2 \pi}}{n}\right)^{3}
$$

или

$$
\frac{n}{n_{2 n}}=\left(\frac{\alpha_{2 n}}{\alpha} \cdot \frac{\xi}{\xi_{2 n}}\right)^{1 / 4} \quad \text { или } \quad \frac{n}{n_{2 л}}=\left(\frac{N u_{2 n}}{N u} \cdot \frac{\xi}{\xi_{2 n}}\right)^{1 / 4}
$$

Формула (12) является критерием эффективности, определяющим отношение числа труб с интенсификаторами к числу гладких труб.

Анализ результатов. Кроме полученной формулы эффективности СВК (12) наиболее конкретным способом определения эффективности СВК является метод сравнения уровня теплообмена в предложенных интенсифицированных [4] гладких трубах. Рассмотрим более подробно его. При выводе формулы эффективности считаются одинаковыми затраты мощности на прокачивание теплоносителя и площадь поверхности теплообмена гладких труб и труб с интенсификацией теплообмена. В этом случае сравниваются коэффициенты теплообмена при полностью развитом течении для трубы с интенсификаторами теплообмена и гладкой трубы.

Запишем эти условия:

$$
\begin{aligned}
N=N_{2 \imath} ; \quad F=F_{2 л} ; \quad d=d_{2 \imath}, & \\
N=\Delta p v=\xi \frac{\ell}{d} \frac{p \omega^{2}}{2} \frac{\pi d^{2}}{4} \omega & =\xi \frac{F}{8} \rho \omega^{3} \\
- & 947-
\end{aligned}
$$


учитывая (13), получим

$$
\xi \frac{F}{8} \rho \omega^{3}=\xi_{2 \pi} \frac{F_{2 \pi}}{8} \rho \omega_{2 \pi}^{3}
$$

или

$$
\begin{aligned}
& \xi \omega^{3}=\xi_{2 \pi} \omega_{2 \pi}{ }^{3}, \\
& \xi \operatorname{Re}^{3}=\xi_{2 n} \operatorname{Re}_{2 n}{ }^{3}
\end{aligned}
$$

откуда

$$
\operatorname{Re}_{2 n}=\left(\frac{\xi}{\xi_{2 n}}\right)^{1 / 3} \mathrm{Re} .
$$

Задаваясь значениями $\mathrm{Re}$, вычисляя $\mathrm{Re}_{\mathrm{rл}}$, можно рассчитать $\mathrm{Nu}$ и $\mathrm{Nu}_{\text {гл. }}$ Если выполняется соотношение

$$
\frac{N u}{N u_{2 \pi}}>1
$$

то поверхность с турбулизаторами считается эффективной.

\section{Выводы}

Вихри, генерируемые выступом, попадают во внешнюю часть внутреннего пограничного слоя и в качестве турбулентности внешнего потока заметно влияют на теплообмен и незначительно на трение стенки.

Интенсификация теплообмена за выступом обеспечивается обновлением пограничного слоя и турбулентностью, создаваемой выступом. Как показывают современные опытные исследования [6], сильное влияние на ранний переход ламинарного пристенного течения в турбулентные оказывает внешняя турбулентность. Шероховатость стенки значительно ускоряет этот переход. Однако учитывая недостаточность сведений о структуре течений и теплообмене за одиночно расположенными выступами шероховатости при малых числах Re, считаем необходимым проведение исследований, подтверждающих интенсификацию теплообмена.

\section{Список литературы / References}

[1] Uzbekov M. O., Tukhtasinov A. G. Thermal efficiency of a solar air-heating collector with a metal chip absorber, J. Sib. Fed. Univ. Eng. \& Technol., 2020, 13(6), 712-720. DOI: 10.17516/1999494X-0260

[2] Шукин В.К. Теплообмен и гидродинамика внутренних потоков в полях массовых сил. М.: Машиностроение, 1970. [Shukin V.K. Heat transfer and hydrodynamics of internal flows in the fields of mass forces. Moscow: Mechanical Engineering, 1970 (in Russian)]

[3] Калинин Э. К., Дрейцер Г.А., Ярхо С.А. Интенсификация теплообмена в каналах. М.: Машиностроение, 1981. [Kalinin E. K., Dreitser G. A., Yarkho S. A. Intensification of heat transfer in the channels. Moscow: Mechanical Engineering, 1981(in Russian)]

$$
-948-
$$


[4] Дрейцер Г.А., Дзюбенко Б. В., Якименко Р.И. Интенсификация теплообмена и анализ методов сравнения теплогидравлической эффективности теплопередающих поверхностей. Тр. Второй Рос. науч. конф. по теплообмену. В. 8 т. Т. 6. Интенсификация теплообмена, радиационный и сложный теплообмен. М.: Издательство МЭИ, 1998, с. 99-102.[Dreitser G.A., Dzyubenko B. V., Yakimenko R. I. Heat transfer intensification and analysis of methods for comparing the thermohydraulic efficiency of heat transfer surfaces. Tr. Second Ros. scientific. conf. on heat exchange. V. 8 t. T. 6. Intensification of heat exchange, radiation and complex heat exchange. M.: Publishing house MEI, 1998, p. 99-102 (in Russian)]

[5] Назмеев Ю. Г., Николаев Н. А. Оценка завихрителей потока, интенсифицирующих процесс теплообмена. ИФЖ, 1979, 4. [Nazmeev Yu.G., Nikolaev N. A. Assessment of flow swirlers that intensify the heat transfer process. IFZh, 1979, 4 (in Russian)].

[6] Кузьма-Кичта Ю. А. Методы интенсификации теплообмена. Учебное пособие. М.: Издательство МЭИ, 2001.[Kuzma-Kichta Yu. A. Methods for intensifying heat transfer. Tutorial. Moscow: MEI Publishing House, 2001(in Russian)].

[7] Митай В.К. Повышение эффективности современных теплообменников. Л.: Энергия, 1980.[Mitai V.K. Improving the efficiency of modern heat exchangers. L.: Energy, 1980 (in Russian)].

[8] Uzbekov M. O. Possibilities of increasing the efficiency of the heat receiver of ics from metal shavings. Computational nanotechnology, 2019, 2, 138-140.

[9] Узбеков М.О., Тухтасинов А.Г. Измерения температуры нагрева абсорбера солнечного воздухонагревательного коллектора. Universum: Технические науки: электрон. научн. журн. 2020, 6(75). URL: http://7universum.com/ru/tech/archive/item/9604 [Uzbekov M.O., Tukhtasinov A.G. Measurements of the heating temperature of the absorber of the solar air heating collector. Universum: Technical sciences: electron. scientific. zhurn. 2020, 6 (75) (in Russian)]

[10] Олимпиев В.В. Ламинарно-турбулентный переход в каналах теплообменников с выступами - интенсификаторами теплообмена. Теплоэнергетика, 2001, 7, 52-56. [Olimpiev V. V. Laminar-turbulent transition in the channels of heat exchangers with protrusions heat exchange intensifiers. Heat Power Engineering, 2001, 7, 52-56 (in Russian)]. 